\title{
A Novel Outlook on the Correlation Between Acute and Chronic Inflammatory States, a Retrospective Observational Study
}

\author{
Seema Mahesh ${ }^{1}$, Mahesh Mallappa ${ }^{2}$, Vitalie Vacaras ${ }^{3}$, Viraj Shah ${ }^{4}$, Elena Serzhantova ${ }^{5}$, \\ Nadezhda Kubasheva ${ }^{6}$, Dmitriy Chabanov ${ }^{5}$, Dionysios Tsintzas ${ }^{7}$, Latika Jaggi ${ }^{8}$, Atul \\ Jaggi $^{8}$, and George Vithoulkas ${ }^{9}$ \\ ${ }^{1}$ Taylor's University \\ ${ }^{2}$ Centre For Classical Homeopathy \\ ${ }^{3}$ Iuliu Hațieganu University of Medicine and Pharmacy \\ ${ }^{4}$ Shah Homeopathic Clinic \\ ${ }^{5}$ Novosibirsk Centre of Classical Homeopathy \\ ${ }^{6}$ Clinic of Nadezhda Kubasheva \\ ${ }^{7}$ General Hospital of Aitoloakarnania \\ ${ }^{8} \mathrm{H} 3$ Centre of Classical Homeopathy \\ ${ }^{9}$ University of the Aegean
}

October 14, 2020

\begin{abstract}
Introduction: Continuum theory states that suppression of efficient acute inflammation is one of the mechanisms responsible for the onset of chronic low-grade inflammation, and in the presence of chronic inflammation, an organism is not capable of an efficient acute inflammatory response to pathogenic stimuli. Materials and methods: We investigated medical records from a large clinical database to assess whether chronic and acute inflammation were mutually exclusive. To evaluate this question, we gathered data on age, current diagnosis, comorbidities and last high fever. Results: A total of 927 cases of chronic inflammatory diseases were investigated. A strong association was found between increasing age and a reduction in concurrent acute and chronic inflammation (chi-squared statistic 51.26; $\mathrm{p}<.00001$ ). Twenty-one individual cases were examined for the pattern of acute and chronic inflammatory diseases. In most cases, there was a clear increase in acute inflammatory conditions as chronic diseases improved. Conclusions: This retrospective study showed a strong association of decreasing concurrent acute and chronic inflammatory states with increasing age, and a possible mutual exclusivity of efficient acute and chronic inflammation was indicated. Since ageing is a low-grade chronic inflammatory process, it is possible that chronic inflammation precludes efficient acute inflammation, which indicates that there is a need to reconsider the manner of handling of acute inflammation in the population.
\end{abstract}

\section{A Novel Outlook on the Correlation Between Acute and Chronic Inflammatory States, a Retrospective Observational Study}

Seema Mahesh ${ }^{* 1,2}$, Mahesh Mallappa ${ }^{2}$, Vitalie Vacaras ${ }^{3}$, Viraj Shah ${ }^{4}$, Elena Serzhantova ${ }^{5}$, Nadezhda Kubasheva $^{6}$, Dmitriy Chabanov ${ }^{5}$, Dionysios Tsintzas ${ }^{7}$, Latika Jaggi ${ }^{8}$, Atul Jaggi ${ }^{8}$, George Vithoulkas ${ }^{9,10}$

Short title: correlation between acute and chronic inflammation

\section{Affiliations:}


$1 *$ Corresponding author: School of Medicine, Faculty of Health and Medical Sciences, Taylor's University, Malaysia

Tel: +60 0123809077; email: bhatseema@hotmail.com

ORCID: 0000-0002-4765-5595

2 Centre for Classical Homeopathy, Bangalore, India

Email: cfchmahesh@gmail.com

ORCID: 0000-0002-8022-4105

3 Department of Neurosciences, "Iuliu Hatieganu" University of Medicine and Pharmacy, Cluj-Napoca, Romania

Email: vvacaras@umfcluj.ro

4 Shah Homeopathic Clinic, Vastrapur, Ahmedabad, India

Email: homeopathvirajshah@gmail.com

5 Novosibirsk Centre of Classical Homeopathy, Novosibirsk, Russia

Email: elenahomeopat@yandex.ru, d.chabanov@mail.ru

6 Clinic of Nadezhda Kubasheva, Moscow Russia, nadia@kubasheva.ru

7 General Hospital of Aitoloakarnania, Agrinion, Greece

Email: dentsin@hotmail.com

8 H3 Centre of Classical Homeopathy, Nashik, India

Email: latikajaggi99@gmail.com, atuljaggi@yahoo.com

9 University of the Aegean, Syros, Greece

10 Postgraduate Doctors' Training Institute, Health Care Ministry of the Chuvash Republic, Cheboksary, Russian Federation

Email: george@vithoulkas.com

ORCID: 0000-0002-9118-8306

Keywords: acute inflammation, chronic inflammation, continuum theory, fever

\section{Summary}

Introduction: Continuum theory states that suppression of efficient acute inflammation is one of the mechanisms responsible for the onset of chronic low-grade inflammation, and in the presence of chronic inflammation, an organism is not capable of an efficient acute inflammatory response to pathogenic stimuli.

Materials and methods: We investigated medical records from a large clinical database to assess whether chronic and acute inflammation were mutually exclusive. To evaluate this question, we gathered data on age, current diagnosis, comorbidities and last high fever.

Results: A total of 927 cases of chronic inflammatory diseases were investigated. A strong association was found between increasing age and a reduction in concurrent acute and chronic inflammation (chi-squared statistic 51.26; $\mathrm{p}<.00001$ ). Twenty-one individual cases were examined for the pattern of acute and chronic inflammatory diseases. In most cases, there was a clear increase in acute inflammatory conditions as chronic diseases improved. 
Conclusions: This retrospective study showed a strong association of decreasing concurrent acute and chronic inflammatory states with increasing age, and a possible mutual exclusivity of efficient acute and chronic inflammation was indicated. Since ageing is a low-grade chronic inflammatory process, it is possible that chronic inflammation precludes efficient acute inflammation, which indicates that there is a need to reconsider the manner of handling of acute inflammation in the population.

\section{Introduction:}

The Continuum of a Unified Theory of Disease proposed the idea that one of the mechanisms responsible for chronic inflammatory diseases is the indiscriminate suppression of efficient acute inflammatory conditions, especially fever with antipyretic drugs [1]. It further posited that in the presence of low-grade chronic inflammation, the organism is incapable of producing efficient acute inflammation in response to pathogenic stimuli. Many immunological studies have provided evidence for the necessity to preserve the acute inflammatory phenomenon in its entirety [2-4]. The mutual exclusion of the two phenomena has been observed in some conditions [5-7], while other studies have used acute inflammation to cure the body of a chronic condition $[8]$.

While Sibley et al. conducted a study to examine the incidence of viral infections in patients with multiple sclerosis and found that multiple sclerosis somehow 'protected' the patients from viral infections [5], no other study, as far as the authors are aware, has investigated chronic inflammatory diseases in general for this phenomenon.

We hypothesised that efficient acute and chronic inflammation are mutually exclusive. We sought to test this by examining the existing clinical data from a private homeopathic medical practice, with the primary objective to investigate whether acute and chronic inflammatory conditions occur concurrently.

\section{Materials and Methods:}

We conducted a retrospective analysis of a large clinical database.

Clinical database: A large clinical case report database from a private homeopathic medical practice was used. The database consisted of over 11,000 case records. The hard copies of the case records were manually inspected for data, and the data were recorded in an Excel sheet. The available data included detailed patient information on demographics, presenting complaints, past medical history, family medical history, laboratory findings, imaging study reports, concurrent medications and homeopathic treatments with follow-ups. The patient information was completely anonymised for the sake of the study, and only the data required for the study were collected.

Eligibility criteria: Patients presenting with chronic inflammatory disease at consultation were included in the study.

Intervention/exposure: We did not consider the effect of any treatment or therapy.

Outcome/measure: The primary outcome of interest for the study was the co-existence of acute and chronic inflammatory states. We also considered different age groups and diagnoses that may have a bearing on the primary outcome.

Here, acute inflammatory conditions was defined as cases with an instance of high fever in the last year prior to the date of consultation.

Exclusion criteria: If the required data were unclear or missing, we excluded the patient and considered only the subjects who had complete data.

Data collected: Data regarding age, sex, current diagnosis, comorbidities, past history, and last high fever were collected.

Statistical analysis: Categorical data are presented as proportions and percentages. 
We performed a chi-squared test to assess the association of acute and chronic inflammatory states. Since the sample population consisted of a wide variety of conditions and diverse age groups, we performed subgroup analysis to test the acute and chronic inflammation association in the different age groups by chi-squared test.

We performed a sensitivity analysis by analysing patients presenting with acute inflammatory conditions at consultation and testing the association of chronic disease in general and with respect to age. $\mathrm{P}<.05$ was considered statistically significant for all the tests.

We further collected cases, from multiple homeopathic practices, of chronic conditions that were improving considerably under treatment and analysed the medical history for patterns of acute and chronic conditions.

Missing data: We did not include any case record where the information was incomplete or unclear. Therefore, no missing data management was carried out.

\section{Results:}

To investigate whether efficient acute and chronic inflammatory states are mutually exclusive, we retrospectively analysed patient case records from a large clinical practice database.

Of the 11,800 case records that were available, 927 chronic inflammatory disease records met the inclusion criteria and were included. There were 393 male $(42.4 \%)$ and 534 female $(57.6 \%)$ patients. The top 5 conditions that emerged were allergies (of all kinds considered together), chronic headaches (including migraine, cluster headache and psychogenic headache), diabetes mellitus, hypertension and chronic fatigue syndrome/post-viral syndrome. Allergic conditions were found to be more commonly associated with acute inflammatory conditions than the other chronic conditions (Fig 1).

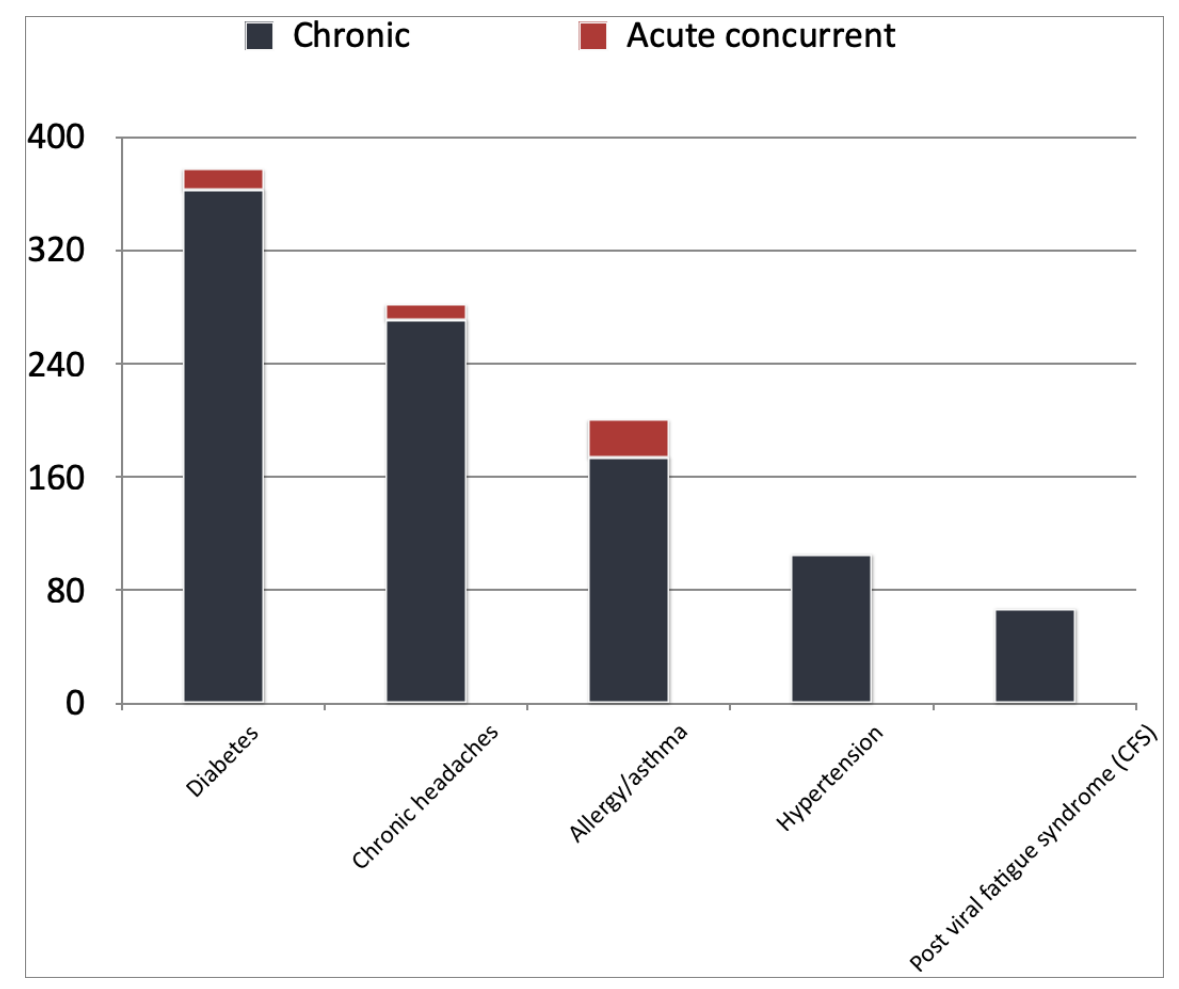

Fig 1. Top 5 chronic conditions in the population and their association with concurrent acute conditions 
Of the 927 chronic inflammatory disease patients, 60 (6.47\%) had concurrent acute inflammatory states, while $867(93.53 \%)$ did not report any history of acute inflammatory disease/high fever in the year prior to consultation (Fig 2).

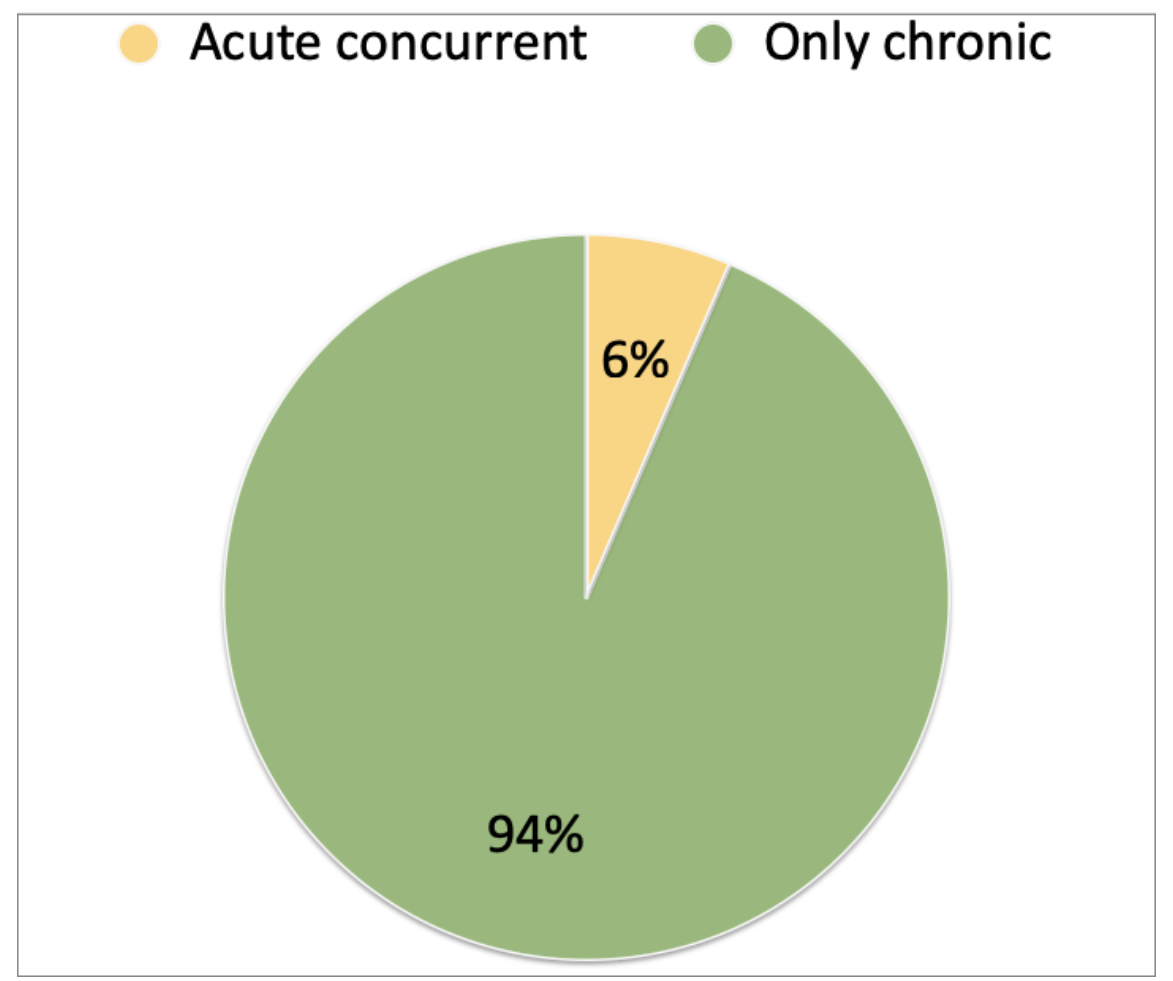

Fig 2. Single chronic conditions with and without acute concurrent states

The population was further divided by age into subgroups, viz., 0 to 17 years, 18 to 64 years and [?]65 years. Table 1 and Fig 3 show the association of acute and chronic inflammatory states in these subgroups. The chi-squared statistic was 51.26; $\mathrm{p}<.00001$, showing a significant association of age with concurrent acute and chronic inflammatory conditions.

Table 1. Age association of chronic conditions with acute concurrent diseases

\begin{tabular}{lllll}
\hline Age group & Chronic only & Acute concurrent & Percentage of concurrent acute & Percentage chronic only \\
\hline $0-17$ years & 113 & 28 & 19.85 & 80.14 \\
$18-64$ years & 647 & 31 & 4.6 & $95.43 ? \dot{\imath} ?$ \\
65 years & 107 & 1 & 0.09 & 99.1 \\
\hline
\end{tabular}




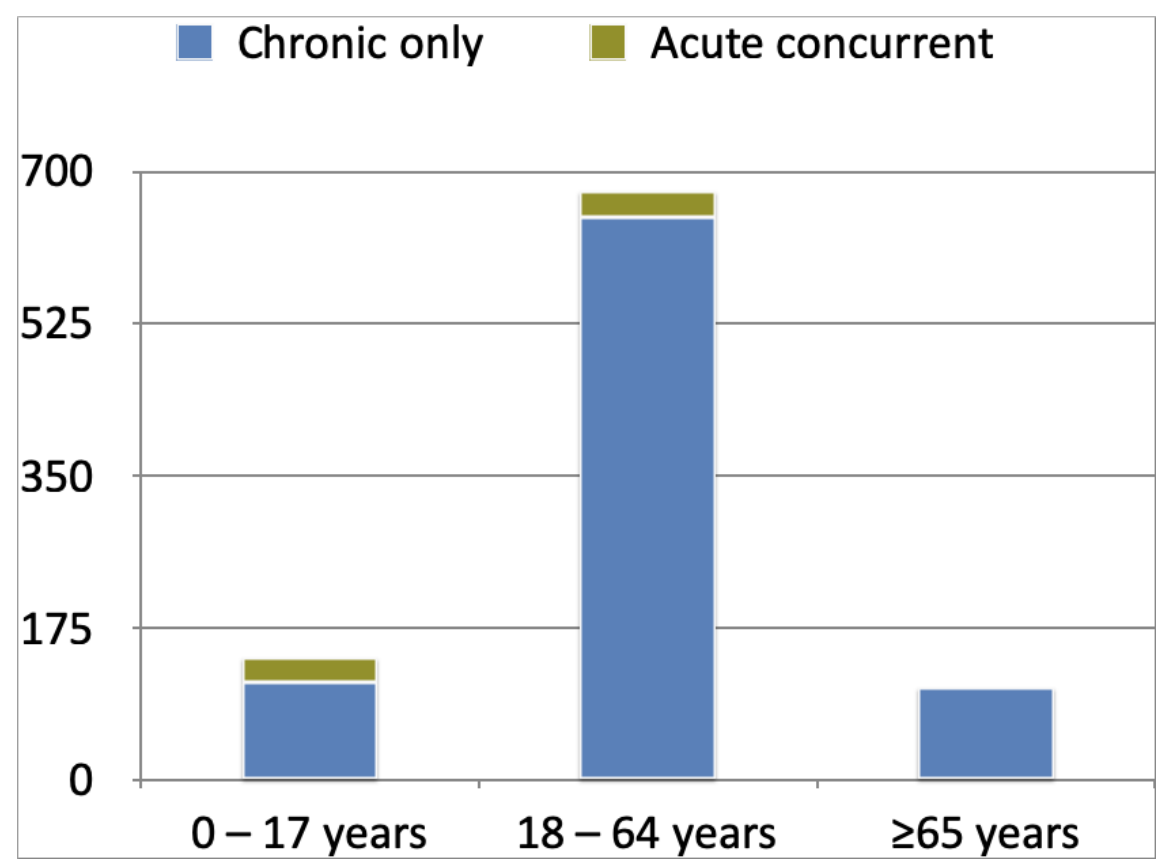

Fig 3. Age association of chronic conditions with acute concurrent diseases

To test the sensitivity of this model, we took a sample of patients that had acute inflammatory disease at presentation and calculated the same measures for concurrent chronic inflammatory disease. We found 69 cases that had complete data and included them. Of the 69 cases, 23 (33.33\%) had concurrent chronic disease, which was very different from the proportion of acute inflammatory disease in the chronic group. These cases were also analysed for the different age groups and conditions, as was done with the chronic group. Table 2 and Fig 4 show the age association with concurrent chronic and efficient acute inflammatory conditions.

Table 2. Age association with concurrent acute and concurrent chronic diseases

\begin{tabular}{lllll}
\hline Age group & Acute only & Chronic concurrent & Percentage of chronic concurrence & Percentage of acute only \\
\hline $0-17$ years & 34 & 4 & 10.52 & 89.47 \\
$18-64$ years & 12 & 17 & 58.62 & $41.38 ? \dot{\imath} ?$ \\
65 years & 0 & 2 & 100 & 0 \\
\hline
\end{tabular}




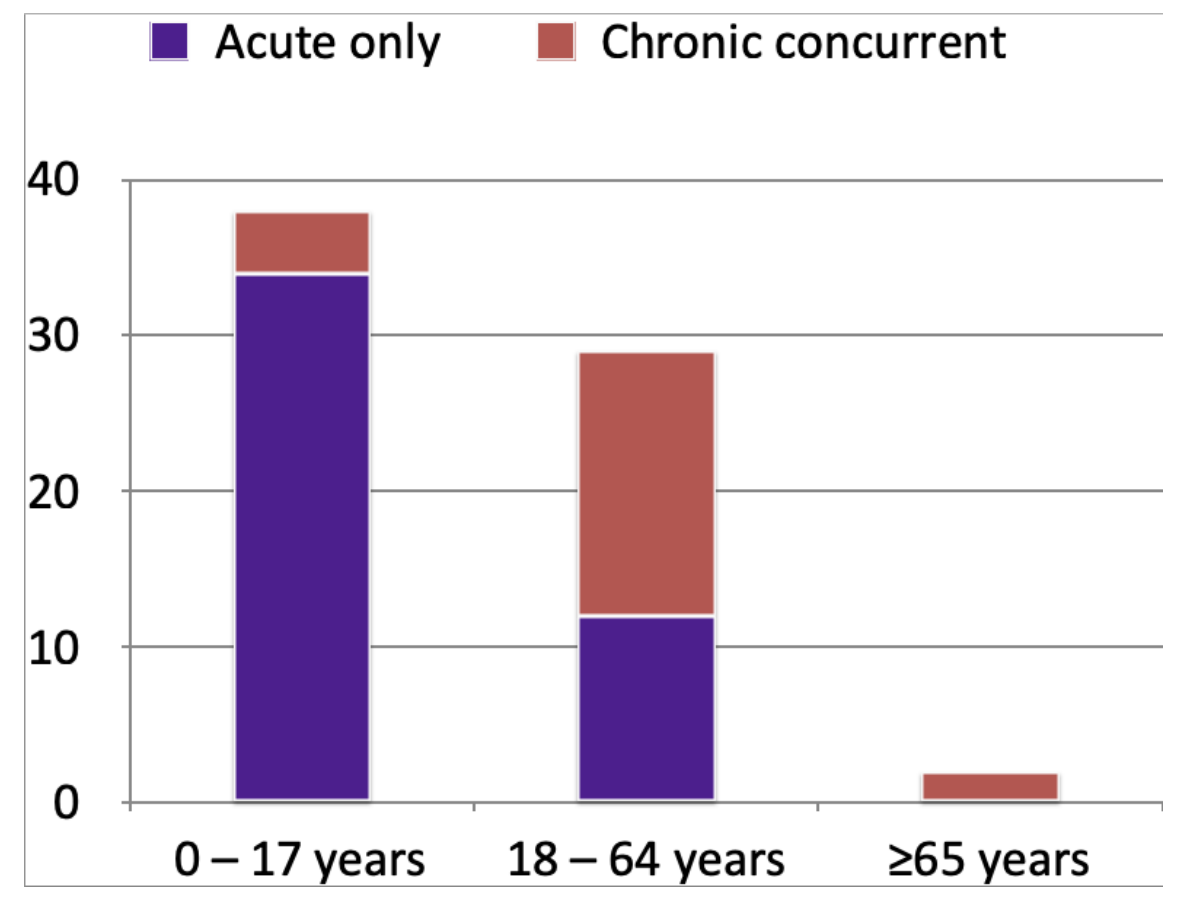

\section{Fig 4. Age association of acute conditions with concurrent chronic diseases}

The trend was similar to that in the chronic group, i.e., with increasing age, the incidence of concurrent acute and chronic inflammatory disease was reduced. Additionally, the same three chronic diseases emerged as those most associated with acute concurrent pathology in this group.

When both these samples were pooled and the chi-squared test was conducted to assess the prevalence of a single inflammatory state as opposed to concurrent states, the analysis showed a strong prevalence of single inflammatory states (chi-squared statistic 60.65; $\mathrm{p}<.00001$ ).

Twenty-one chronic conditions that improved considerably under treatment were mapped for the pattern of acute and chronic inflammatory conditions (supplementary material). For this mapping, the frequency (episodes of acute inflammatory diseases) and relative severity (e.g., fever temperature, severity of chronic symptoms, and the Childhood Autism Rating Scale - as applicable) of these conditions were analysed over the course of the individual's history. In 18 cases, there was a clear increase in acute inflammatory conditions as the chronic disease showed improvement; however, prior to the onset of chronic disease, many cases showed repeated acute inflammatory diseases, which did not occur while the chronic disease was active. There was another interesting pattern in two cases, where previously suppressed skin conditions re-emerged as the chronic neurological conditions improved. According to homeopathic principles, this is the best evidence for improvement of a condition that is considered to be deeper than the skin (such as neurological conditions) $[1,9,10]$. The discussion of this phenomenon, however, is not within the scope of this paper.

\section{Discussion:}

The global disease burden has steadily shifted from acute infections to noncommunicable diseases, most of which are chronic inflammatory in nature [11]. Continuum theory states that one of the reasons is the manner in which acute diseases, especially high fever, are handled at the population level [1].

Efficient acute inflammation is the hallmark of a healthy immune system and is characterised by high fever [1]. Fever is essential for the cellular and humoral components of host defence to be recruited into action against pathogens - a systemic alarm [4, 12]. Once recruited, these cells and chemical factors (e.g., cytokines and leukotrienes) go through a series of tightly orchestrated events whose ultimate goal is not 
just neutralisation of the pathogen but also restoration of tissue homeostasis [3, 13-15]. In the absence or malfunction of any of these checkpoints, inflammation does not resolve [2]. Failure to resolve inflammation has been discovered as one of the main mechanisms in the onset of chronic inflammation [16]. This would mean that the activated, persistent, low-grade inflammation will not have the same level of response as that seen in an immunologically intact organism. The response will either be absent/very minimal or exaggerated in a dangerous sense when the virulence of the pathogen is too high. In neither case will the response be efficient or optimal. According to the levels of health theory, those people with moderately severe chronic illnesses will react in an exaggerated way to a pathogenic challenge, while those with severe/terminal diseases may not respond to it at all [10], a fact that was emphasized in the recent pandemic of COVID-19 [17].

Furthermore, the phenomenon of ageing itself is a chronic inflammatory process $[18,19]$. This implies that chronic inflammatory diseases increase with age and that concurrent acute and chronic inflammation must decrease accordingly. With this background, we must investigate whether the prevalence of chronic disease presupposes the absence of efficient acute inflammation. While immunological experimental studies are required to establish the mechanisms of this hypothesis, this study aimed to investigate whether such a phenomenon might be present in the population.

Our study showed that not only did the incidence of chronic diseases increase with age, but the concurrent acute and chronic inflammatory diseases decreased significantly with age. Allergic conditions, which, according to the levels of health theory, are some of the more superficial and less serious chronic diseases, were most associated with acute states when compared to the more serious chronic inflammatory disorders, such as chronic fatigue syndrome and hypertension. These diseases are more serious and more difficult to treat and cure homoeopathically than allergies.

Furthermore, the analysis of individual disease patterns in 21 patients indicated that efficient acute and chronic inflammation were likely mutually exclusive. In the sensitivity analysis, there was a strong pattern of decreased concurrent acute and chronic inflammation with age in this sample population as well. Considering ageing to be a chronic inflammatory process, our hypothesis is supported by our findings.

The study has a few limitations. We did not include case records that had incomplete data, and their bearing on the outcome cannot be ruled out; however, the sample size was still sufficiently large. We did not consider the effect of treatment or therapy of any kind in this study, and it will be interesting to examine this pattern in those with a history of treatment compared to those without to rule out the actual effect of drugs. The database is from a homeopathic medical practice that is sought mostly by people who have not found satisfactory results with conventional medicine, creating selection bias. This bias may be overcome by conducting the same analysis on medical records from a public hospital. The final limitation is the accuracy of the fever history given by patients. While homeopathic case documentation involves recording the minutest details regarding a patient's health, there can still be discrepancies due to recall bias in the patient. This can be overcome by performing a longitudinal study and following people's health status over time.

\section{Conclusions}

In a retrospective study of a large clinical database, we evaluated concurrent efficient acute and chronic inflammatory conditions. Our study showed a significant decrease in the concurrence of inflammatory disease with increasing age, suggesting a possible mutual exclusivity between the two inflammatory states. Further immunological studies are necessary to understand this mechanism. Meanwhile, it may be beneficial to reconsider the treatment of acute inflammatory conditions in the population to minimize the burden of chronic inflammatory disease.

Funding : None

Conflict of interest: The authors declare there are no conflicts of interest.

Ethical clearance: Not applicable (secondary data from clinical records) 
Consent for participation: Not applicable

Consent for publication: Not applicable

Availability of data and materials: from Mendeley: http://dx.doi.org/10.17632/c7zyt694f2.1

Authors' contributions: SM and MM analysed the data for the retrospective study from the large clinical database. SM, MM, VV, VS, ES, NK, DC, DT, LJ and AJ were the primary physicians who treated, analysed and provided the data for the 21 additional cases presented here. SM wrote the manuscript and did the referencing. GV is the guarantor and guide of this study. All authors have read the final version and approved the manuscript.

The poster of this study was presented at the 'Movementis' conference in TelAviv, Israel in July 2019.

Acknowledgements: The authors thank Anjum Warsi, Amritha Belagaje and Pooja Dhamodar for technical assistance.

\section{References}

1. Vithoulkas G, Carlino S. The "continuum" of a unified theory of diseases. Med Sci Monit $2010 ; 16: 15$.

2. Serhan CN, Savill J. Resolution of inflammation: the beginning programs the end. Nat Immunol $2005 ; 6$ :1191-7.

3. Rajakariar R, Yaqoob MM, Gilroy DW. COX-2 in inflammation and resolution. Molecular interventions $2006 ; 6: 199$.

4. Evans SS, Repasky EA, Fisher DT. Fever and the thermal regulation of immunity: the immune system feels the heat. Nature Reviews Immunology 2015; 15 :335-49.

5. Sibley W, Bamford C, Clark K. Clinical viral infections and multiple sclerosis. The Lancet $1985 ; \mathbf{3 2 5}$ :1313-5.

6. Torres AR. Is fever suppression involved in the etiology of autism and neurodevelopmental disorders? BMC Pediatrics 2003; $3: 9$.

7. Megremi ASF. Is fever a predictive factor in the autism spectrum disorders? Medical Hypotheses 2013; $80: 391-8$.

8. Hobohm U. Fever therapy revisited. Br J Cancer 2005;92 :421-5.

9. Hahnemann S. Organon of medicine: B. Jain publishers, 2002.

10. Vithoulkas G. Levels of health. 3rd revised edition

Edn. Alonissos, Greece: International Academy of Classical Homeopathy, 2019.

11. GBDCoD C. Global, regional, and national age-sex-specific mortality for 282 causes of death in 195 countries and territories, 1980-2017: a systematic analysis for the Global Burden of Disease Study 2017.Lancet $2018 ; 392$ :1736-88.

12. Fisher DT, Vardam TD, Muhitch JB, Evans SS. Fine-tuning immune surveillance by fever-range thermal stress. Immunol Res 2010;46 :177-88.

13. Wallace JL. COX-2: A Pivotal Enzyme in Mucosal Protection and Resolution of Inflammation. TheScientificWorldJOURNAL 2006;6 :941592.

14. Barnig C, Bezema T, Calder PC, Charloux A, Frossard N, Garssen J, Haworth O, Dilevskaya K, LeviSchaffer F, Lonsdorfer E, Wauben M, Kraneveld AD, Te Velde AA. Activation of Resolution Pathways to Prevent and Fight Chronic Inflammation: Lessons From Asthma and Inflammatory Bowel Disease. Frontiers in immunology 2019; 10 :1699-. 
15. Loynes CA, Lee JA, Robertson AL, Steel MJ, Ellett F, Feng Y, Levy BD, Whyte MKB, Renshaw SA. PGE2 production at sites of tissue injury promotes an anti-inflammatory neutrophil phenotype and determines the outcome of inflammation resolution in vivo. Science Advances 2018; 4 :eaar8320.

16. Lawrence T, Gilroy DW. Chronic inflammation: a failure of resolution? International journal of experimental pathology 2007;88:85-94.

17. Wang B, Li R, Lu Z, Huang Y. Does comorbidity increase the risk of patients with COVID-19: evidence from meta-analysis. Aging 2020;12:6049-57.

18. Franceschi C, Bonafe M, Valensin S, Olivieri F, De Luca M, Ottaviani E, De Benedictis G. Inflammaging: An Evolutionary Perspective on Immunosenescence. Annals of the New York Academy of Sciences 2000; $908: 244-54$.

19. Ginaldi L, Loreto MF, Corsi MP, Modesti M, De Martinis M. Immunosenescence and infectious diseases. Microbes and Infection 2001; 3 :851-7. 